\title{
Individualization of psychosocial problems and the alternative
}

\begin{abstract}
In recent years, many psychological problems are defined as illnesses and then medicated. Mental health professionals seem to redefine social, cultural and societal problems as individual diseases. The individualization and psychologization of psychosocial problems leads to more patients in mental health services and a virtually insatiable treatment demand. In this remark, we are dealing with the causes of this development and account for a psychosocial, contextual model, an alternative to the biomedical, individual model.
\end{abstract}

Keywords: psychosocial problems, mental disorders, medicalization
Volume 2 Issue I - 2018

\author{
Arnulf Kolstad \\ Department of Psychology, University of Science and Technology, \\ Trondheim, Norway \\ Correspondence: Arnulf Kolstad, Department of Psychology, \\ University of Science and Technology, Trondheim, Norway, \\ Email arnulf.kolstad@ntnu.no
}

Received: February 14, 2018 | Published: March 02, 2018

\section{Introduction}

What are the reasons that social and societal problems are increasingly being understood and treated as psychological and individual diseases? What consequences does it have for individuals, societies and the public health? Why are relationship problems rewritten to individual diagnoses? What are the alternatives? These are the topics of this comment. Psychiatry and psychology is not alone in focusing on individual's body and brain. The individual human beings, detached from relationships and responsibilities in endeavor to realize themselves, have become an ideal in the Western, individualistic culture. Psychiatry and psychology contribute to the individualization with focusing on individual diagnoses. This means of understanding seeping in everywhere and helping to characterize men's thinking and understanding of themselves. One of the consequences is more patients in the mental health services. Never has it in Western countries been as many patients as now. ${ }^{1}$ This is the exhortation in an individualistic culture where relationships and 'we-identity' crumble and are portrayed as second-rate. The self-perception is characterized by this cultural descent and we ask for a diagnosis to get some sort of explanation for the discontent, restlessness and emptiness. It becomes more common to define own difficulties as sickness. It is at the doctors and in psychiatry the help apparently exists and the implication is more patients in mental health care. The biological trend in psychiatry that characterizes the thinking of causal conditions and treatment modalities contributes undoubtedly to individualization and increased drug use. More time-consuming and patient centered treatment methods are downgraded and given confined conditions. Social circumstances, poverty, the senseless or hard work are not causes of mental illness in the biomedical discourse. The genes and the brain are to blame for personal misery. To find happiness in life, the individual must restore the chemical balance of the brain, ie. Increase the serotonin level, according to biological psychiatry. ${ }^{1,2}$

\section{From psychosocial problems to individual disease}

There are many examples of the inclination to redefine social and relational issues to problems located in the brain or in the genes. The work life's wear is rewritten to the individual case of illness. Children's problems at school and home are medicated due to the individual diagnosis of ADHD. Alternative causal explanations for unrest, lack of concentration and learning disabilities are losing in the competition with a biological oriented psychiatry/psychology that sees human problems primarily as flaws in the biological machinery. It is bad enough that the psychology helps to camouflage cultural flaws and societal problems as individual failures, worse it becomes when such also become biological failures. Poor mental health is largely due to the impact people are exposed to. Epidemiological studies have shown that the poor are overrepresented in almost all disease categories. ${ }^{3}$ Weak economy leads to deficient mental health. The poor cannot afford to live a 'normal' and decent life and receive a negative self-esteem of dignity and shame. ${ }^{4}$ Poverty and loneliness are therefore more important and banal causes of deprived mental health than laziness, lack of morality, chemical imbalance in neurotransmitters or other 'machine faults'. The poor do not have anomalies in the brain more frequent than rich people do. ${ }^{5}$ The poor people's problems are due to their life experiences and not lack of chemicals in the brain or what kind of genetic equipment they have Societal and cultural relationships are repressed in a medicalized diagnostic discourse on individual pathology. ${ }^{5}$ Focusing on genetic and brain failures helps to camouflage cultural burden, and biographical and individual mistakes are being considered as biological errors. The language of the biomedical psychiatry says implicitly that this biology is not good enough, and assuming we find methods, we should intervene in and improve it in the brain and body.

Psychiatric illnesses cannot be diagnosed with urine- or blood samples. Psychiatrists must deal with people's personal perception 
and presentation of their problems. Most people perceive their problems as relational and social. It is therefore important to maintain social and societal conditions such as poverty, unemployment, poor living conditions, social exclusion, stigma and social isolation as explanations for human behavior, for thoughts, emotions, and health. Especially if we want to have a dialogue with the patients. A cultural and social understanding of people, and thus of their psychology, emphasizes that joys and problems are sociocultural conditional or mediated and cannot be teared off from, but must be understood in a cultural context. ${ }^{6}$ Dysfunctional and bothersome thoughts, emotions, and relationships have their roots in the way people relate to each other. In individualistic cultures with an emphasis on autonomy, competition and a successful façade, it becomes increasingly difficult to establish and maintain healthy and equal relationships. It creates outsiders or losers who do not hold out in the rat race, enforced out of work and have problems with self-respect and self-esteem. Psychiatrists and psychologists focus more and more on the "individual offerings" and the need for tailored therapy in accordance with an increasingly detailed diagnostic manual that makes 20 percent of the people in the western world psychiatric patients. The daily life's difficulties and dissatisfaction cannot however, be repaired with ever more mental health effort. The discomfort is not a regrettable result of an otherwise well-functioning society, but it is the logical consequence of a culture based on individual competition and cultivating the autonomous, 'free' human being. Psychologization and its harmful consequences cannot be removed without altering the society that builds on and promotes ever-stronger individualism and competition.

\section{A psychosocial model}

A psychosocial model of psychiatric disorders assumes that individual problems and disorders primarily relate to the relationship between people and to the environment in broad sense. Such problems and disorders have as little as other thoughts and feelings encountered by themselves inside the body of a human being. They occur because of what we have experienced and acquired since conception, and especially after birth. ${ }^{7}$ By preserving this perspective on mental illnesses, acknowledging brain plasticity and human ability to change, we can also counteract the suffering. By changing behaviors, environment, influencing thoughts and feelings in a deliberate and appropriate manner, painful and traumatic, often unworked and nonexpressed emotions and thoughts, can change. This change will also show up in the neural network of the brain and thus stick to both the mind and the biology. ${ }^{8}$ Such change of consciousness and the brain will not have any adverse effects as other interventions in the brain, such as shock therapies, lobotomy and chemotherapy.

\section{The efficacy of psychotherapy}

In his book The Great Psychotherapy Debate, Bruce Wimped (2001) takes up the issue of what characterizes the impactful psychotherapy. Based on research, including several meta-analyses, he discusses two models with various scientific and methodological approaches: The medical model and the contextual model. The medical model, focusing on the single individual, argues that the therapist's medical expertise is most important for the outcome of the therapy: how closely the manual or treatment protocol for a particular diagnosis is followed. The contextual model is dealing with the relationship to or alliance with the patient, not focusing on a particular diagnosis. The research findings show that treatment based on a medical model is a poor solution when dealing with psychiatric disorders. The 'therapeutic alliance' is a key factor for the outcome - regardless of the technique used. The alliance means more than all specific therapy factors. Who the therapist is, his or her qualities are more vital for the outcome than following a manual, and personal traits may be more important than formal education. The most effective therapists are according to those considered by the patients, even after a few meetings, as good helpers and which form part of an alliance with the clients. The most effective or helpful therapists had a more psychological and a less biological approach to the clinical process. The best therapists also believed that therapy should take place over a longer period than the therapists who had worse results. The medical model focusing on individual diagnosis provides according to inferior treatment results, and he suggests that it is important to free the treatment of psychiatric problems and disorders from the medical establishment's links. ${ }^{9}$ Discarding the medical model has major implications for the health care and for the education and training of therapists.

\section{Acknowledgement}

None

\section{Conflict of interest}

Authors declare there is no conflict of interest towards this manuscript.

\section{References}

1. American Psychiatric Association (APA). Let's Talk Facts About Depression; 2005.

2. Moncrieff J. The Chemical Imbalance Theory of Depression: still promoted but still unfounded. 2014.

3. Kolstad A. Epistemology of Psychology-A New Paradigm: The Dialectics of Culture and Biology. Hauppauge NY. 2013.

4. Kolstad A. The Nature-Nurture Problem Revisited. Some Epistemological Topics in Contemporary Human Sciences. Open Journal of Philosophy. 2013;3(4):517-521.

5. Underlid K. Poverty and health - Reflections and empirical discoveries. Vardøger. 2006;(30):114-127.

6. Ekeland TJ. Biology as Ideology Vardøger. 2006;(30):65-84.

7. Kolstad A. Inter-Functionality Between Mind, Biology and Culture: Some Epistemological Issues Concerning Human Psychological Development. In: Maria Lucia, Seidl-de-Moura, editors. Human Development-Different Perspectives. 2012;(2):19-41.

8. Kolstad A. From the machine paradigm to brain plasticity and how culture overrules biology in humans. Psychology. 2012;3(9):691-697.

9. Wampold BE. The Great Psychotherapy Debate. Models, Methods and Findings. London; 2001. 